\title{
A NOTE ON HERMITIAN OPERATORS ON FUNCTION SPACES
}

\author{
TOSHIKO KOIDE
}

(Communicated by Palle E. T. Jorgensen)

\begin{abstract}
In this note we shall get concrete expressions of hermitian operators on a closed subspace of $C(\Omega)$ which contains constant functions and separates points of $\Omega$.
\end{abstract}

\section{INTRODUCTION}

Let $X$ be a complex Banach space, and let $T$ be a bounded linear operator on $X . T$ is said to be hermitian if its spatial numerical range $V(T)$ is real. It is known that $T$ is hermitian if and only if $\|\exp (i t T)\|=1$ for all real $t$. Hermitian operators are interesting objects in operator theory. For basic properties of the spatial numerical range and hermitian operators, the reader is referred to $[2,3]$.

Let $B(X)$ be the algebra of all bounded operators on $X$, and let $H(X)$ be the set of all hermitian operators on $X$. In [2] it is shown that $B(X)=$ $H(X)+i H(X)$ if and only if $X$ is a Hilbert space. This is a very useful fact, and we can know the certain character of complex Banach spaces by examining hermitian operators on them. Hermitian operators on various complex Banach spaces were investigated by many authors. Especially, in [1] Berkson showed that the spaces $A C([0,1]), C^{(1)}([0,1]), \operatorname{Lip}([0,1])$, and $\operatorname{lip} \alpha, 0<\alpha<1$, admit only trivial hermitian operators, that is, real multiples of the identity operator by using the expression of linear isometries $[8,13]$. In $[8,13] \mathrm{Rao}$, Roy, and de Leeuw obtained concrete expressions of linear isometries on the above four concrete spaces by embedding them into certain closed subspaces of $C(\Omega)$. Thus, in this note we shall get concrete expressions of hermitian operators for an arbitrary closed subspace of $C(\Omega)$ which contains constant functions and separates points of $\Omega$.

First, we shall state several definitions and notation. Let $\Omega$ be a compact Hausdorff space, and let $C(\Omega)$ be the Banach space of all complex-valued continuous functions on $\Omega$ with the supremum norm. The closed subspace $M$ of $C(\Omega)$ is called a function space if it contains constant functions and separates points of $\Omega$. For a function space $M$ let $\operatorname{Ch}(M)$ be the Choquet boundary, and let $\Gamma_{M}$ be the Silov boundary. Denote by $K(M)$ the set of all $L$ in $M^{*}$

Received by the editors May 27, 1993.

1991 Mathematics Subject Classification. Primary 46E15, 47B15, 47B38.

Key words and phrases. Hermitian operators, function spaces, closed unbounded *-derivation. 
such that $L(1)=1=\|L\|$. For $x \in \Omega$, let $\Phi(x)$ be the element of $K(M)$ defined by $(\Phi(x))(f):=f(x) \quad(f \in M)$. Then $\Phi$ is a homeomorphism from $\Omega$ into $K(M)$ with $w^{*}$-topology.

Let $M$ be a function space, and let $T$ be a surjective linear isometry of $M$. The concrete expression of $T$ was investigated by Novinger [9] and Okayasu and Takagaki [10]. In [9] Novinger showed that $T$ is expressed by a continuous mapping $h_{0}$ of $\operatorname{Ch}(T(M))$ onto $\mathrm{Ch}(M)$. However, since $\mathrm{Ch}(T(M))$ is not necessarily closed and $h_{0}$ cannot be necessarily extended to $\Gamma_{T(M)}$, his result is not available for our purpose. On the other hand, in [10] Okayasu and Takagaki proved the following theorem.

Theorem A. Let $M$ be a function space of $C(\Omega)$, and let $T$ be a linear isometry of $M$ into $C(\Omega)$. Then there exist a closed boundary $\Gamma$ of $T(M)$, a continuous mapping $h$ of $\Gamma$ onto $\Gamma_{M}$, and a scalar-valued function $w$ defined on $\Omega$ with $|w(x)|=\|w\|_{\infty}=1 \quad(x \in \Gamma)$ such that

$$
(T(f))(x)=w(x) f(h(x)) \text { for all } f \in \text { Mand } x \in \Gamma \text {. }
$$

Since $\Gamma$ is closed in compact space, this theorem is available for our purpose. Now, we shall show that hermitian operators are represented by a multiplication operator and a closed unbounded *-derivation.

\section{MAIN ReSUlt}

Theorem. Let $M$ be a function space of $C(\Omega)$, and let $H$ be a hermitian operator on $M$. Suppose $H(1) M \subseteq M$. Then there exist a real-valued continuous mapping $h$ on $\Omega$ and a closed unbounded *-derivation $D$ in $C\left(\Gamma_{M}\right)$ such that

$$
\left.H(f)\right|_{\Gamma_{M}}=\left.h f\right|_{\Gamma_{M}}-i D\left(\left.f\right|_{\Gamma_{M}}\right) \text { for all } f \in M \text {. }
$$

Remark. If $M$ is an algebra, an assumption $H(1) M \subseteq M$ is satisfied. Also many spaces consisting of functions satisfy this assumption.

The proof is divided into three steps.

Since for each real $t \exp (i t H)$ is a surjective linear isometry of $M$, it follows from Theorem A that there exist a closed boundary $\Gamma$ of $T(M)$, a surjective continuous mapping $h_{t}$ of $\Gamma$ onto $\Gamma_{M}$, and a scalar-valued function $w$ defined on $\Omega$ with $|w(x)|=\|w\|_{\infty}=1 \quad(x \in \Gamma)$ such that

$$
(\exp (i t H)(f))(x)=w(x) f\left(h_{t}(x)\right) \text { for all } f \in M \text { and } x \in \Gamma \text {. }
$$

If $H(1)=0$, then $\exp (i t H)(1)=1$ for each real $t$. Hence $w(x)=1$ for all $x \in \Gamma$. And if $\Gamma_{M}=\Omega$, we have $\Gamma=\Omega$. Therefore, if the above two assumptions hold, then there exist a surjective continuous mapping $h_{t}$ of $\Omega$ and a scalar-valued function $w$ defined on $\Omega$ with $|w(x)|=\|w\|_{\infty}=1 \quad(x \in \Omega)$ such that

$$
(\exp (i t H)(f))(x)=f\left(h_{t}(x)\right) \quad \text { for all } f \in M \text { and } x \in \Omega .
$$

Now, we first suppose $H(1)=0$ and $\Gamma_{M}=\Omega$.

Step 1. Suppose $H(1)=0$ and $\Gamma_{M}=\Omega$.

Lemma 1. $\left\{h_{t}\right\}_{t \in R}$ has the following three properties.

(1) $h_{t+s}=h_{t} \circ h_{s}$ and $h_{0}$ is the identity mapping.

(2) Each $h_{t}$ is a homeomorphism of $\Omega$.

(3) For each $x \in \Omega, h_{t}(x)$ is continuous with respect to $t$. 
Proof. (1) is clear. For (2) and (3), since

$$
(\exp (i t H))^{*}(\Phi(x))=\Phi\left(h_{t}(x)\right) \quad \text { for all } x \in \Omega
$$

from $(*)$, we have

$$
h_{t}(x)=\Phi^{-1}(\exp (i t H))^{*}(\Phi(\dot{x})) \text { for all } x \in \Omega .
$$

Therefore, (2) and (3) hold.

For each real $t$, we define $S_{t}$ by

$$
\left(S_{t}(g)\right)(x):=g\left(h_{t}(x)\right) \text { for all } x \in \Omega \text { and } g \in C(\Omega) .
$$

Then we have the following lemma.

Lemma 2. $\left\{S_{t}\right\}_{t \in R}$ is a strongly continuous one-parameter group of *automorphisms of $C(\boldsymbol{\Omega})$.

Proof. Since it is clear that $\left\{S_{t}\right\}_{t \in R}$ is a one-parameter group of *-automorphisms of $C(\Omega)$, we show $\left\{S_{t}\right\}_{t \in R}$ is strongly continuous, that is,

$$
\lim _{t \rightarrow 0}\left\|S_{t}(g)-g\right\|_{\infty}=0 \text { for all } g \in C(\Omega) .
$$

Since if $\left\{S_{t}\right\}_{t \in R}$ is a one-parameter group, it is strongly continuous if and only if it is weakly continuous, that is,

$$
\lim _{t \rightarrow 0} F\left(S_{t}(g)-g\right)=0 \text { for all } F \in C(\Omega)^{*} .
$$

Thus we may show that $\left\{S_{t}\right\}_{t \in R}$ is weakly continuous. By Riesz's representation theorem, Lebesgue's bounded convergence theorem, and Lemma 1, we have

$$
\begin{aligned}
\lim _{t \rightarrow 0} F\left(S_{t}(g)-g\right) & =\lim _{t \rightarrow 0} \int_{X}\left(S_{t}(g)-g\right)(x) d \mu(x) \\
& =\lim _{t \rightarrow 0} \int_{X}\left(g\left(h_{t}(x)\right)-g(x)\right) d \mu(x) \\
& =\int_{X} \lim _{t \rightarrow 0}\left(g\left(h_{t}(x)\right)-g(x)\right) d \mu(x)=0 .
\end{aligned}
$$

Therefore, $\left\{S_{t}\right\}_{t \in R}$ is strongly continuous.

Let $D_{0}$ denote the generator of $S_{t} . D_{0}$ is a closed unbounded *-derivation from Lemma 2. Since

$$
S_{t}(f)=\exp (i t H)(f) \text { for all } f \in M,
$$

the domain of $D_{0}$ is a ${ }^{*}$-algebra which contains $M$ and

$$
D_{0}(f)=\left.\frac{d}{d t} S_{t}(f)\right|_{t=0}=i H f \text { for all } f \in M .
$$

Therefore, we have $H=-\left.i D_{0}\right|_{M}$.

Step 2. Next, we consider the case that $H(1)=0$ and $\Gamma_{M} \subseteq \Omega$. Denote by $\widetilde{M}$ the restriction of $M$ to $\Gamma_{M}$. 
Lemma 3. The operator $\phi:\left.f \rightarrow f\right|_{\Gamma_{M}}$ is an isometric isomorphism between $M$ and $\widetilde{M}$. Moreover, $\widetilde{M}$ is a function space with $\Gamma_{M}=\Gamma_{\widetilde{M}}$.

Proof. We show that $\Gamma_{M}=\Gamma_{\widetilde{M}}$. Since $\widetilde{M} \subseteq C\left(\Gamma_{M}\right)$ and given the definition of $\widetilde{M}, \Gamma_{\widetilde{M}} \subseteq \Gamma_{M}$. Next we show $\Gamma_{M} \subseteq \Gamma_{\widetilde{M}}$. Since $\left\|\left.f\right|_{\Gamma_{M}}\right\|_{\infty}=\|f\|_{\infty}$ and given the definition of $\Gamma_{\widetilde{M}}$, for arbitrary $f \in M$ there exists a point $x \in \Gamma_{\widetilde{M}}$ such that

$$
\left|\left(\left.f\right|_{\Gamma_{M}}\right)(x)\right|=\left.|| f\right|_{\Gamma_{M}} \|_{\infty}
$$

which means that $|f(x)|=\|f\|_{\infty}$. Since $\Gamma_{\widetilde{M}}$ is a closed boundary for $M$, we have $\Gamma_{M} \subseteq \Gamma_{\widetilde{M}}$. Therefore, $\Gamma_{M}=\Gamma_{\widetilde{M}}$.

We define a linear operator $\widetilde{H}$ of $\widetilde{M}$ by

$$
\widetilde{H}\left(\left.f\right|_{\Gamma_{M}}\right):=\left.H(f)\right|_{\Gamma_{M}}=\phi H \phi^{-1}\left(\left.f\right|_{\Gamma_{M}}\right) \text { for all } f \in M \text {. }
$$

Then $\widetilde{H}$ is a hermitian operator on $\widetilde{M}$. Therefore, it follows from Lemma 3 and Step 1 that there exists a closed unbounded *-derivation $D$ in $C\left(\Gamma_{M}\right)$ such that $\widetilde{H}=-\left.i D\right|_{\widetilde{M}}$. Therefore, we have

$$
\left.H(f)\right|_{\Gamma_{M}}=\tilde{H}\left(\left.f\right|_{\Gamma_{M}}\right)=-i D\left(\left.f\right|_{\Gamma_{M}}\right) \quad \text { for all } f \in M \text {. }
$$

Step 3. In order to complete the proof, we consider general cases.

Lemma 4. $H(1)$ is a real-valued continuous function on $\Omega$.

Proof. $H(1)\left(x_{0}\right)=\left(\Phi\left(x_{0}\right)\right)(H(1)) \in V(H)$ for all $x_{0} \in \Omega$. Since $H$ is a hermitian operator on $M, V(H)$ is real. Therefore, $H(1)$ is a real-valued continuous function on $\Omega$.

Set $h:=H(1)$. Define $L_{h}$ by

$$
L_{h}(g):=h g \text { for all } g \in M \text {. }
$$

Then $H-L_{h}$ is a hermitian operator on $M$ and $\left(H-L_{h}\right)(1)=0$. Therefore, it follows from Step 2 that there exists a closed unbounded *-derivation $D$ in $C\left(\Gamma_{M}\right)$ such that

$$
\left.\left(H-L_{h}\right)(f)\right|_{\Gamma_{M}}=-i D\left(\left.f\right|_{\Gamma_{M}}\right) \quad \text { for all } f \in M .
$$

Therefore, there exist a real-valued continuous mapping $h$ on $\Omega$ and a closed unbounded *-derivation $D$ in $C\left(\Gamma_{M}\right)$ such that

$$
\left.H(f)\right|_{\Gamma_{M}}=\left.h f\right|_{\Gamma_{M}}-i D\left(\left.f\right|_{\Gamma_{M}}\right) \text { for all } f \in M \text {. }
$$

\section{ACKNOWLEDGMENT}

The author would like to express her heartfelt thanks to Professor Seiji Watanabe for his valuable suggestions and advices.

\section{REFERENCES}

1. E. Berkson and A. Sourour, The hermitian operators on some Banach spaces, Studia Math. 52 (1974), 33-41.

2. F. F. Bonsall and J. Duncan, Numerical ranges of operators on normed spaces and of elements of normed algebras, London Math. Soc. Lecture Note Ser., vol. 2, Cambridge Univ. Press, London, 1971. 
3. F. F. Bonsall and J. Duncan, Numerical ranges II, London Math. Soc. Lecture Note Ser., vol. 10, Cambridge Univ. Press, London, 1973.

4. M. Cambern, Isometries of certain Banach algebras, Studia Math. 25 (1965), 217-225.

5. M. Cambern and V. D. Pathak, Isometries of spaces of differentiable functions, Math. Japon. 26 (1981), 253-260.

6. N. Dunford and J. T. Schwartz, Linear operators Part I: General theory, Interscience, New York, 1958.

7. K. Jarosz and V. D. Pathak, Isometries between function spaces, Trans. Amer. Math. Soc. 305 (1988), 193-206.

8. K. de Leeuw, Banach spaces of Lipschitz functions, Studia. Math. 21 (1961), 55-66.

9. W. P. Novinger, Linear isometries of subspaces of continuous functions, Studia Math. 53 (1975), 273-276.

10. T. Okayasu and M. Takagaki, Linear isometries of function spaces, RIMS. Kôkyûroku (Kyoto Univ.) 743 (1991), 130-140. (Japanese)

11. V. D. Pathak, Linear isometries of absolutely continuous functions, Canad. J. Math. 34 (1982), 298-306.

12. R. R. Phelps, Lectures on Choquet's theorem, Van Nostrand, Canada, 1966.

13. N. V. Rao and A. K. Roy, Linear isometries of some function spaces, Pacific J. Math. 38 (1971), 177-192.

14. A. K. Roy, Extreme points and linear isometries of the Banach spaces of Lipschitz functions, Canad. J. Math. 20 (1968), 1150-1164.

15. K. W. Tam, Isometries of certain function spaces, Pacific J. Math. 31 (1969), 233-246.

Department of Mathematical Science, Graduate School of Science and Technology, NiIgATA UNIVERSITY, NiIgATA, 950-21, JAPAN 